Manuscript received June 11, 2014; accepted for publication December 30, 2014; published online January 15, 2015

Department of Textile, Ghent Univ., 9052 Ghent, Belgium (Corresponding author), e-mail: benny.malengier@ugent.be

2 Laboratory of Geotechnics, Ghent Univ., 9052 Ghent, Belgium,

e-mail: gemmina.diemidio@ugent.be

3 Laboratory of Geotechnics, Ghent Univ., 9052 Ghent, Belgium,

e-mail: herman.peiffer@ugent.be

${ }^{4}$ Department of Industrial System and Product Design, Ghent Univ., Campus Kortrijk, G. K. De Goedelaan 5, 8500 Kortrijk, Belgium,

e-mail: Cristina.Ciocci@UGent.be

Faculty of Mathematics, Physics and Informatics, Comenius Univ. Bratislava, Mlynská dolina, 84248 Bratislava, Slovakia,

e-mail: pavol.kison@gmail.com
Benny Malengier, ${ }^{1}$ Gemmina Di Emidio, ${ }^{2}$ Herman Peiffer, ${ }^{3}$ Maria-Cristina Ciocci, ${ }^{4}$ and Pavol Kišon ${ }^{5}$

\section{Unsaturated Permeability and Retention Curve Determination From In-Flight Weight Measurements in a Bench-Scale Centrifuge}

\section{Reference}

Malengier, Benny, Di Emidio, Gemmina, Peiffer, Herman, Ciocci, Maria-Cristina, and Kišon, Pavol, "Unsaturated Permeability and Retention Curve Determination From In-Flight Weight Measurements in a Bench-Scale Centrifuge," Geotechnical Testing Journal, Vol. 38, No. 2, 2015, pp. 243-254, doi:10.1520/ GTJ20140121. ISSN 0149-6115

\section{ABSTRACT}

Centrifuges have been used in many ways to determine permeabilities, as they can significantly shorten testing times in low-permeable soils. Typically equilibrium profiles, inflow-outflow measurements, or direct measurements from inside the sample-like from tensiometers or radioactive decay-are used. Recently, weight measurements of the sample outside the centrifuge were used effectively in an adapted setup. We present the results of the possibilities offered by doing transient weight measurements of a soil-sample during rotation in the centrifuge. We present the setup of such an experiment and how the unsaturated permeability and water retention curve can be recovered from it. This eliminates the overhead of doing measurements inside the sample.

\section{Keywords}

centrifuge, unsaturated flow, unsaturated permeability, water retention curve

\section{Introduction}

Knowing the permeability of soils is important to achieve effective soil use; for example, in the construction of infiltration zones, or of covers for waste dumps. Also when drainage is applied to lower the groundwater level, it is indispensable to know saturated and unsaturated permeabilities in the planning phase.

Determining the saturated hydraulic conductivity is traditionally done with a flexible wall permeameter, while the water retention curve (also called soil-water characteristic curve, SWCC) and the unsaturated permeability are determined via a combination of techniques, of which the 
pressure plate setup, ASTM D2325-68(2000), now part of ASTM D6836-02(2008)e2, (ASTM 2008) is most used for the type of grounds used in this study. A consequence of this is that often two different samples of the same soil are used in determining the permeabilities. The pressure plate experiment needs 2 to 3 months, leading to a very slow turn-over capacity.

The quality of a pressure plate experiment typically depends on the number of data points, and often 5 or 6 points are taken. Without prior knowledge of the SWCC, selecting these pressure points can be suboptimal in obtaining the correct SWCC, as the 6 chosen pressure points at which to determine the saturation might be placed such that the important part of the water retention curve, where there is fast saturation change, is missed. Taking more data points increases the duration of the experiment, so time must be balanced with quality.

Several alternative, faster methods were developed to determine the SWCC, but they are not yet commonly used in the field. One is based on using centrifugation to speed up the drainage process. This was initiated by Hassler and Brunner (1945). A detailed overview on the development of this method can be found in several articles (Nimmo 1990; Nimmo et al. 2002; Van den Berg et al. 2009) and the citations therein. Nimmo et al. (1991) showed that steady-state unsaturated flow is possible in a centrifuge, allowing for the performance of accurate outflow experiments using equilibria values. This is, however, still a very slow technique. For example, the unsaturated flow apparatus (UFA) method is sometimes used (Conca and Wright 1998; Khaleel et al. 1995) to determine unsaturated permeability. Steady-state can be achieved in hours; however, this must be done for several experimental data points. Zornberg and McCartney (2010) developed a centrifuge permeameter allowing the determination of the SWCC and the hydraulic conductivity from steady state flow regimes.

Due to performant computer codes, it became possible in recent decades to use transient data (Caputo and Nimmo 2005; Šimůnek and Nimmo 2005). The centrifuge permeameter for transient infiltration was investigated by Parks et al. (2012). Parameter identification based on transient data might allow the extraction of conductivity data in a couple of hours.

It quickly became clear that only using cumulative outflow data of transient flow does not always lead to correct results (Firoozabadi and Aziz 1991; Nakajima and Stadler 2006). As a consequence, the cumulative outflow data is typically extended with at least one pressure measurement from inside the sample; see, e.g., McCartney and Zornberg (2010). An alternative is to use electrical conductivity (Nimmo 1990; Šimůnek and Nimmo 2005). A point of attention with tensiometers is that they might show a delay in registering the outside capillary heads, especially when due to centrifugation there are fast changes.

An alternative approach is the simplified evaporation method as used by Peters and Durner (2008), suitable for the wet to moderately dry parts of the water retention curve. This method also requires the use of two tensiometers inside the sample, but is already much faster than a pressure plate experiment.

More recently attempts have been done to adapt existing bench-scale centrifuges to determine SWCC and hydraulic conductivity, instead of designing custom centrifuges. Martins Reis et al. (2011) showed this to be successful in a steady-state fixed flow setup. Kišon et al. (2013) showed that measuring the gravitational center (GC) of the unsaturated sample outside the small centrifuge at prescribed times gives sufficient extra information to attempt the determination of the relative permeability. This simplifies the sample preparation considerably, as one no longer needs to obtain a measurement from inside the sample. In this approach, the centrifuge needs to be stopped at regular intervals, and weight measurements must be performed. This has the advantage that the measurements can be very precise, but has as the disadvantage that a lot of sample handling is needed and introduces errors.

The objective of this study was to demonstrate it is possible to determine the permeability in the wet range with a benchscale centrifuge setup that measures the weight of the sample tube (MT) and the weight of the cumulative outflow during centrifugation (MO). The testing method is very user friendly: Load the sample in the centrifuge (no use of tensiometers required), start the data logging and centrifugation, and determine soil parameters via the developed software. The weight of the sample measurement serves here as the replacement of the pressure measurement in a point of the sample. It is the analogue of doing a GC measurement outside of the centrifuge, as done by Kišon et al. (2013).

All outflow methods have the problem that the dry range is very difficult to investigate. In order to investigate the dry region, outflow experiments (standard multistep-outflow or centrifuge drainage) are best combined with evaporation experiments to investigate the entire range, see, e.g., Schelle et al. (2011). The method described in this paper will extrapolate the results to the dry region, but this extrapolation should not be assumed correct on face value.

We next present the materials and centrifuge method used and three experimental runs with the centrifuge. Our main contribution is showing that the technique is viable, and in identifying technical challenges that need to be overcome to obtain an easy to use and accurate bench scale centrifuge to obtain the water retention curve and unsaturated permeability. The technique is usable for all ground types that are sufficiently rigid.

\section{Materials and Methods}

\section{RICHARDS EQUATION}

When considering unsaturated flow (hydraulic head $h<0$ ), the Richards equation must be solved. In the case saturated zones 
$(h \geq 0)$ are also present, this must be combined with Darcy's law. For one dimensional flow they are given by

(1) $\begin{cases}\partial_{r} q=0, \quad q=-K_{s}\left[\partial_{r} h-\frac{\omega(t)^{2}}{g} r\right], & h \geq 0 \\ \partial_{t} \theta=K_{s} \partial_{r}\left[k(\theta)\left(\partial_{r} h-\frac{\omega(t)^{2}}{g} r\right)\right], & h<0\end{cases}$

where:

$q=$ the fluid flow,

$\theta=$ the saturation of the porous media,

$r=$ the distance from the centrifuge axis,

$\omega(t)=$ the angular speed of rotation (in radians per second),

$K_{s}=$ the saturated hydraulic conductivity,

$g=$ the gravitational constant, and

function $K_{s} k(\theta)=$ the hydraulic conductivity in the unsaturated region, with $k(\theta)$ the relative permeability.

The initial condition for an experimental run (after measurements) is the original saturation $\theta$ of the sample where unsaturated, and $h(r, 0)=0$ otherwise (as $\omega[0]=0$ ). Efficient solvers are available, like Hydrus-1D. We used the solver as described by Kačur et al. (2011) and Kišon et al. (2013).

To be able to solve the Richards equation, one needs knowledge of the saturated hydraulic conductivity $K_{s}$, and two soil hydraulic properties, the SWCC $\theta(h)$ and the relative permeability $k(\theta)$. In this paper, we reduce the complexity considerably by reducing the problem of determining these two unknown functions to determining only two unknown parameters. We do this by considering soil hydraulic properties, which were proposed by van Genuchten (1980)

(2)

$$
\begin{aligned}
S_{e} & =\frac{1}{\left(1+(\gamma h)^{n}\right)^{m}}, \quad h \in(-\infty, 0) \\
k\left(S_{e}\right) & =S_{e}^{1 / 2}\left[1-\left(1-S_{e}^{1 / m}\right)^{m}\right]^{2}
\end{aligned}
$$

where:

$m=1-1 / n, n>1$ and $\gamma$ are empirical soil parameters, and

$\left.S_{e}=\left(\theta-\theta_{r}\right) / \theta_{s}-\theta_{r}\right)=$ the effective saturation, where $\theta_{s}$ is the volumetric water content at saturation and $\theta_{r}$ the residual volumetric water content.

The disadvantage of this approach is that soils that do not satisfy the van Genuchten SWCC (Eq 2) cannot be correctly characterized by the method. However, this will be evident by bad matching of model results with the experimental data. Note, however, that this is the reason why extrapolated permeability values cannot be trusted. There is ample evidence that this van Genuchten-Mualem approach often leads to good matches if only part of the unsaturated region is investigated, but breaks down if the sample goes from completely dry to fully saturated, see, e.g., Schelle et al. (2011).

Our approach can be summarized as follows: The sample undergoes drainage according to the Richards equation. This leads to outflow that is measured by the outflow weight sensor,
$M O(t)$. The drainage leads to a specific saturation distribution over the sample, which relates to a gravitational center and moves away from the center of the sample. The sample tube weight sensor measures this weight as $M T(t)=r_{G C}(t) M(t) \frac{\omega^{2}}{g}$, where $M$ is the mass of the sample tube, $r_{G C}$ is the gravitational center, and $g$ is the standard gravity. As the only unknowns in the Richards equation are $K_{s}, n$, and $\gamma$, an inverse procedure can be applied to find those model parameter values that produce $M O(t)$ and $M T(t)$ values that best match the actual measurements. The code used by Kišon et al. (2013) was extended for the case presented in this paper and is available. ${ }^{6}$

As it is with normal multistep-outflow experiments, one often starts with a saturated experiment (so called extended multistep-outflow), so as to determine $K_{s}$ accurately and independently. We will also do this and give the results of a $K_{s}$ determination. Next, the same sample is used to determine the water retention curve and relative permeability. For this, the parameters $\theta_{s}$ and $\theta_{r}$ are also required. They need to be determined with existing experimental methods; although setting $\theta_{r}=0$ is a possibility here as the very dry region is not sampled.

\section{CENTRIFUGE INSTRUMENTATION}

The bench-scale centrifuge used for the experiments is a type Sigma 3-18. This setup is equipped with two microprocessors for the independent control of the rotor recognition and the over-speed signal. The speed is continuously controlled by these microprocessors with an accuracy of $1 \mathrm{rpm}$ and a maximum speed of $4000 \mathrm{rpm}$. The duration of centrifugation and acceleration and deceleration curves can be set and were determined during the experiments by filming the centrifuge display readout during acceleration and deceleration of the rotor.

The centrifuge is provided with four buckets. Uneven loading of oppositely located buckets may lead to imbalance; in that case, the drive automatically switches off and an imbalance warning message is displayed. For this reason, the bucket containing the soil sample is carefully weighed with the aim to obtain similar total weights (with an accuracy of $1 \mathrm{~g}$ ). The maximum radius from the central rotor to the base of the buckets is $171 \mathrm{~mm}$.

The soil sample is housed in a wind protected closed system, which permits free movement of air into and out of the soil sample where a constant air pressure prevails (see Fig. 2). Hence we can assume the pore air pressure in the sample to be atmospheric.

The closed centrifuge bucket contains a glass container which can be screwed to the top of the centrifuge bucket. This glass container is constituted by a Duran glass tube (LSB Inner tube, inner diameter $21.55 \mathrm{~mm}$ by height $85 \mathrm{~mm}$ ) containing the soil sample. The soil container tube is provided with a

${ }^{6}$ See public code repository https://gitorious.org/centrifuge-1d/ 
FIG. 1 Engineering drawing of the centrifuge bucket with sensors. S1: Top tension load cell sensor (mass tube measurement, MT), S2: Base subminiature compression sensor (ass outflow measurement, MO), F: Filter embedded in the glass container hanging on S1, M: Ground sample, C: Outflow cup resting on S2.

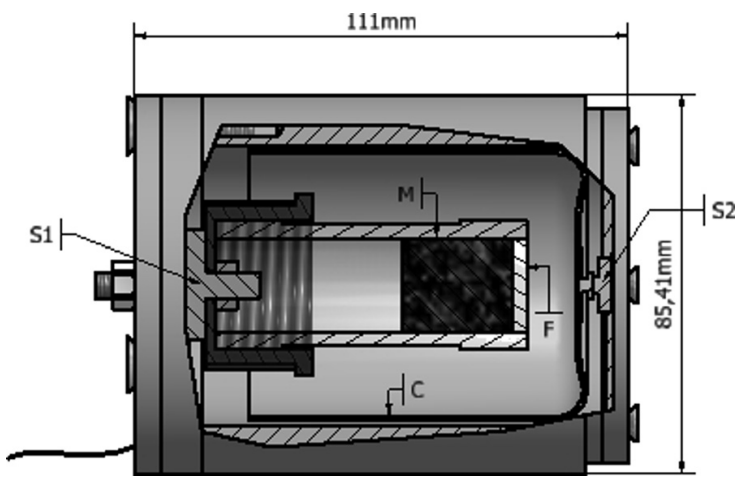

Borosilicate glass 3.3 Vitra POR filter welded to the tube with a nominal pore size $1-1.6 \mu \mathrm{m}$ (class 5 , ISO $/ 4793=\mathrm{P} 1,6$ ), thickness from 2.55 to $3 \mathrm{~mm}$, and a diameter of about $21.55 \mathrm{~mm}$. The glass containers are provided of a sufficiently thick wall $(3 \mathrm{~mm}$, sufficient to withstand the lateral expansion under the horizontal stresses) resistant to centrifugal forces. Air holes are present at the top of the glass container so as to permit free air movement in the top section.

The necessary dimensions (e.g., height of the soil) were measured using a Vernier caliper (accuracy $0.01 \mathrm{~mm}$ ). The mass of the soil, inlet, and outlet water were measured by means of laboratory scales (accuracy $0.01 \mathrm{~g}$ ) at start and end of a centrifuge run. The measurements in flight were done with load cell sensors. For the measurement of the water mass at the outlet cup, an Omega compression load cell was selected, type subminiature LCMKD compression, with high accuracy. For the determination of the centrifugal force (necessary for the determination of the center of gravity) a tension load cell sensor from Omega, type subminiature LCMFD tension with high accuracy, was chosen. The load cells were built into a container fitting in the centrifuge bucket; see Fig. 1 for an engineering drawing of the load cell and Fig. $\mathbf{2 ( b )}$ for a picture of the load cell.

The load cells were connected to an external data acquisition unit via a slip ring. A slip ring is an electromechanical device that allows the transmission of power and electrical signals from a stationary to a rotating structure. SenRing SNM012A-24S used here can be used in any electromechanical system that requires unrestrained, intermittent, or continuous rotation while transferring power or data. Two types of slip ring were used for this research: A slip ring with brushes suitable for rotational speeds between 600 and $1000 \mathrm{rpm}$ and a slip ring with gold-to-gold contact suitable for rotational speeds higher than $1000 \mathrm{rpm}$ of the order of $2000 \mathrm{rpm}$. The metal contacts of the second type are maintenance free and are supposed to provide long life.

To avoid noise due to the high speed rotation of the slip ring, miniature amplifiers from Synectic were added (SY0143WL, 3 wire 4-20 mA 9-20 V supply low voltage $2 \mathrm{mV} / \mathrm{V}$ Full scale). The full schematic setup is shown in Fig. 3. During the experiments it was noticed that isolation of the amplifiers was needed due to contact with the metallic part of the centrifuge (such as the rotating arm).

The load cell sensors are calibrated with known weights, which were constructed out of plastic. This allowed for the correct conversion of the voltage measured into the weight of the samples in the centrifuge. Every pair of sensors used was suitable for a different range of capacities in order to be able to reach the required accuracy for the different rotational speeds needed in order to obtain a complete water retention curve with the continuous measurements. In Fig. 3(b), we show a picture of the fully assembled testing rig.

\section{MATERIALS}

The materials used for this investigation are a mixture of $10 \%$ kaolin and $90 \%$ sand, as well as undisturbed samples from

\section{FIG. 2}

(a) The glass container with a soil sample inside placed on balances to determine initial value of the gravitational center of the sample. (b) Sample holder parts: Base subminiature compression sensor (bottom left) for the determination of the outle mass, subminiature top tension load cell sensor (bottom right) for the measurement of the weight of the sample under the centrifugal force, and the centrifuge holder containing our custom centrifuge bucket.

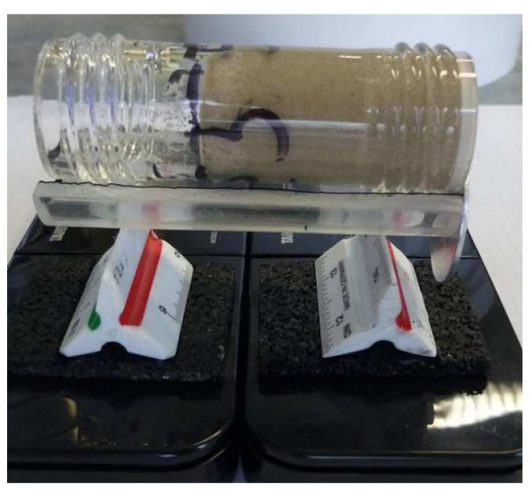

(a)

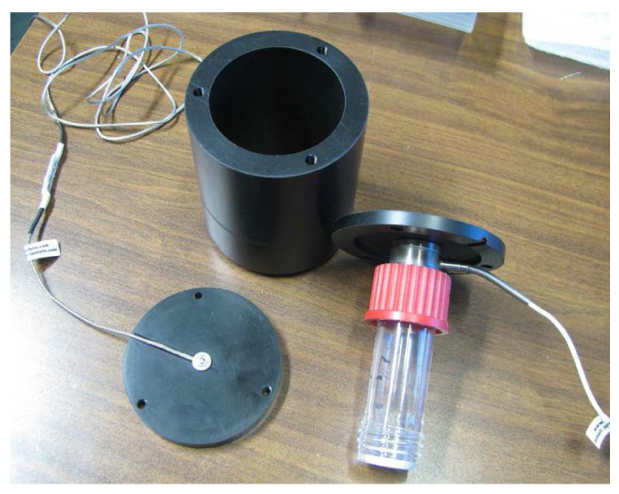

(b) 
FIG. 3

(a) Schematic representation of the Sigma 3-18 centrifuge as used. $\mathrm{B}$ is the bucket in which the custom centrifuge bucket (Fig. 1) is placed, C the counterweight, A the amplifier for the measurement signal, $\mathrm{S}$ the slip ring, DAQ the acquisition unit which is placed outside the centrifuge. (b) Actual equipped centrifuge, in the front bucket the black centrifuge bucket is visible and the counterweight bucket in the back

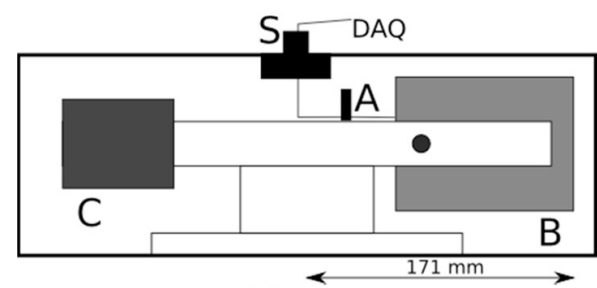

(a)

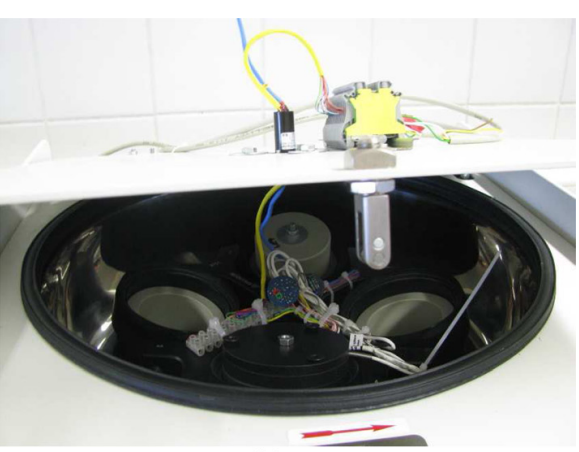

(b)
Zwijnaarde, a village in Belgium, which consist of sand with silty clay. The mixtures of kaolin clay and sand chosen, following the reference (Chiu and Shackelford 1998), were particularly useful because the arising soil parameters are representative of typical soil mixtures encountered in the field above the water table, showing a suitable low hydraulic conductivity (due to the presence of some \% of clay that close the pores of the sand), but is sufficiently stiff (due to the presence of the sand) to avoid problems of excessive compressibility of the sample during centrifugation. These characteristics allowed for the validation of the model (where a constant height is required).

The mixtures of kaolin and sand were prepared as follows: Both clay and sand were dried in the oven at $105^{\circ} \mathrm{C}$ for $16 \mathrm{~h}$. With a ceramic mortar and pestle, some clods of clay were crushed. The clay was then sieved with a $63 \mu \mathrm{m}$ sieve. The clay and the sand were mixed thoroughly. From there, $90 \mathrm{~g}$ of dry Mol sand (a fine quartz sand) with $10 \mathrm{~g}$ of dry kaolin clay were mixed with a spoon. The soil was then placed in the tube and water was poured from the top to saturate the sample under $1 \mathrm{~g}$ conditions until the visible water front reached the base filter. The initial water content of the mixture was about $20 \%(\mathrm{~kg} / \mathrm{kg})$. We identify these samples as CSM-1, CSM-2, and CSM-3.

The undisturbed samples were taken above the water level in a site in Zwijnaarde, Belgium (see Fig. 4). These samples were analyzed with the standard methods for the determination of the water retention curve in parallel with the instrumented centrifuge. We identify these sample as ZW-1 up to ZW-10.

\section{TRADITIONAL MEASUREMENTS}

We performed falling head tests on the samples to determine the saturated permeability. The porosity $\theta_{s}$ was also determined and an ASTM D2325 test was conducted to determine the water retention curve. This provides 5 data points, as seen in Fig. $\mathbf{5}$, and nonlinear regression is used to fit the van Genuchten parameters to this curve. The results are given in Table 1. Values determined via a method that produces error margins are indicated with their error. Here, CSM-1 is the sample on which
ASTM D2325 (pressure plates) was performed, CSM-2 were the results from Kišon et al. (2013), and CSM-3 was the sample created for the new tests. The different ZW samples are samples from three different bore holes at different depths, which shows the quick variation that is possible in retention curves between samples which are close by.

To better understand the obtained variation for the Zwijnaarde samples, we show the resulting water retention curves for the different samples in Fig. 6.

ZW-01 and ZW-02 are from the same borehole and match ZW-07 and ZW-08 relatively well from a different borehole, although ZW-08 deviates at lower water content. ZW-09 and ZW-10 from a third borehole are qualitatively different. All tests with the centrifuge were executed with samples from the first two boreholes.

\section{DATA PROCESSING}

The measurement data $M O(t)$ and $M T(t)$ are two time series measurements of volts as measured by the sensors, which are converted to weights according to a predetermined calibration curve. Next, the tare must be subtracted from the measurement to obtain the actual influence of the water mass. A high scatter in the measurement data was observed; this scatter was probably due to several effects such as: Slip ring noise, precess of the axis of rotation, unbalance effects, ability of the centrifuge to maintain a fixed rotational speed, and accuracy of the sensors.

An inverse method like Levenberg-Marquardt (LM) can work with scattered data provided all data points are included, and provided data points carry equal weights. In this experiment, however, we will have a start with a sharp change (typically up to $3 \mathrm{~min}$ ), and then a slow change towards an equilibrium (20-60 min). If all measurement points are included in the inverse determination with equal weight, the slow change towards equilibrium will completely shadow the sharp change, leading to bad results. To alleviate this, one can change the weights given to experiment points, or one can reduce the points used in the inverse method. We opted for the last. This, in turn, 
FIG. 4 Ground extraction device. (a) Ground probe to collect a sample. (b) A sample ring embedded in a larger sample. (c) Transfer of the sample ring into the glass tube. (d) Close up of a prepared glass tube with ground sample present, and filter paper placed on top.

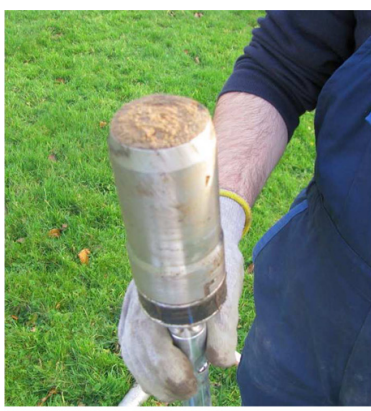

(a)

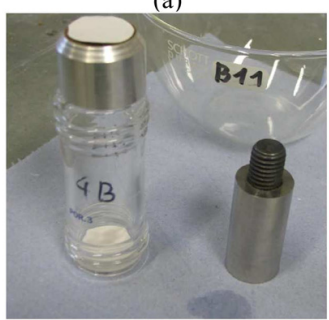

(c)

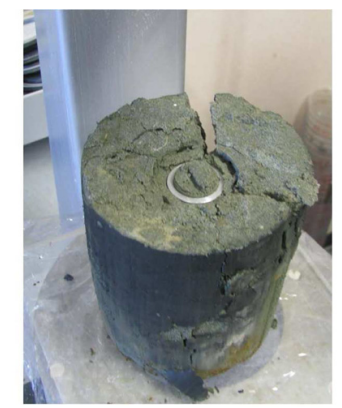

(b)

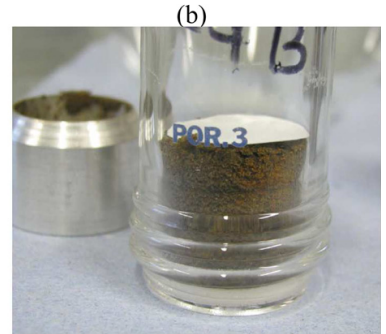

(d) requires that the measurements are smoothed, so that selection of a measurement can be done without need to take the scatter into account.

For the slowly changing signal during rotation, a Gaussian rolling window average was used as smoothing. This was combined with an outlier detection test (Bonferroni test on rolling window ordinary least squares results) to remove the occasional bad data.

\section{Experimental Results}

In the tests in this study, a ground sample first underwent a number of centrifuge runs in saturated conditions, in which water was poured on the sample, and the centrifuge was used to generate saturated flow. These runs serve a double purpose. First, they allow one to determine the saturated conductivity. Secondly, they allow one to verify that the ground sample retains a fixed height under centrifugation at the required rotational speeds. After the saturated runs, a centrifuge run with an unsaturated specimen was performed. The height of the sample and outflow weight was always measured before and after a run. Mass balance was verified to guarantee no evaporation losses occurred. If the height of the sample during an unsaturated run changed more than $1 \%$, the test was rejected and another ground sample was used. This was because the transient drainage model assumes constant sample height, and gravitational center determination requires homogeneous density in the sample.
FIG. 5 SWCC of ZW-01. 5 data points obtained with ASTM D2325 as crosses, and the resulting water retention curve obtained via curve fitting.

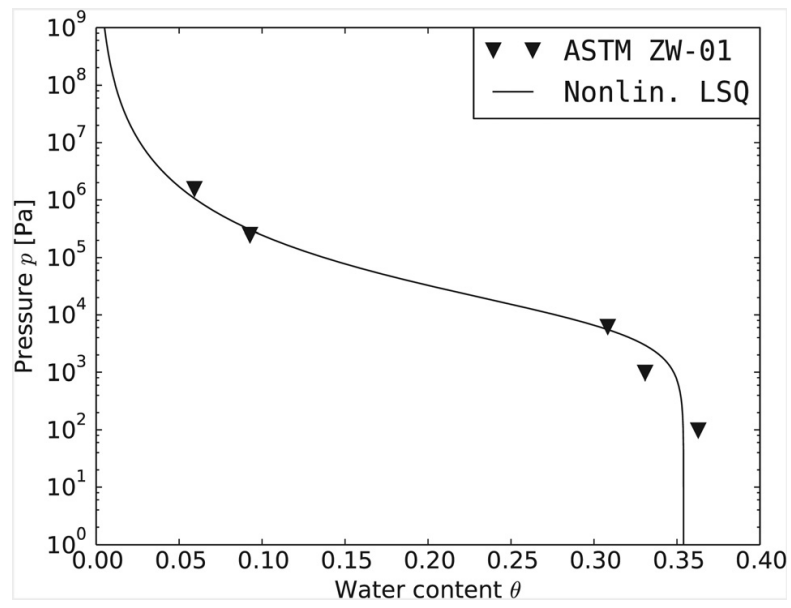

\section{CLAY-SAND MIXTURE}

In Kišon et al.'s (2013) work, a clay-sand mixture (CSM) was tested with a centrifuge, measuring outflow water and gravitational center at different rotational speeds after prescribed durations (the start-stop result). From this, the saturated and relative permeability was determined. A new clay-sand mixture was created in the same manner (CSM-3), and tested with the method developed here at $600 \mathrm{rpm}$. As seen in Table 1, the different CSM samples have saturated permeability that vary from $10^{-7}$ to $3 \times 10^{-6}$, with the sample tested being the most permeable, $K_{s}=2.9 \times 10^{-6} \mathrm{~m} / \mathrm{s}$. This variation might also lead to a water retention curve that does not match the values obtained on CSM-1 and CSM-2.

The sample was centrifugated at $600 \mathrm{rpm}$ for $2 \mathrm{~h}$. The results when using all measurements- only the water weight in the sample (MT) or only the water outflow weight (MO)-are given in Table 2. The root mean square (RMS) errors of the inverse solution and the covariance matrix of the LM-method indicated a valid minimum was found.

The determined saturated conductivity of $K_{s}=2.4 \times 10^{-6}$ $\mathrm{m} / \mathrm{s}$ is comparable to the traditional measurement in Table 1. The unsaturated tests also give comparable values for the $n$ parameter, but a higher $\gamma$ value. To better understand the differences in the recovered parameters, we visually show the result in Fig. 7. In Figs. $\mathbf{7 ( a )}$ and $\mathbf{7}(\boldsymbol{b})$, we see the measured values for $\mathrm{MO}$ and MT compared to the simulated results when using only the MO measurement, only the MT measurement, or both measurements. These results are given in Newton force contribution of the water. The three obtained results are all acceptable, as seen in Table 2, when compared with Table 1. However, the sample weight measurement does not fully complement the outflow weight measurement. This indicates that, in the sample, the gravitational center migrates slower towards the base of the sample with a given outflow than what is obtained from using 
TABLE 1 Results of traditional measurements of $K_{S}, \theta_{S}$, and water retention curve, on 9 samples. CSM samples are $10 \%$ kaolin, $90 \%$ fine quartz sand mixtures, ZW samples are sand with silty clay from an undisturbed site in Belgium.

\begin{tabular}{|c|c|c|c|c|}
\hline Sample & $K_{s}(\mathrm{~m} / \mathrm{s})$ & $\gamma\left(\mathrm{kPa}^{-1}\right)$ & $n$ & $\theta_{s}$ \\
\hline CSM-1 & $2.110^{-6}$ & $-0.183 \pm 0.060$ & $2.18 \pm 0.53$ & $0.368 \pm 0.035$ \\
\hline CSM-2 & $(0.11 \pm 0.12) 10^{-6}$ & $-0.132 \pm 0.036$ & $1.99 \pm 0.37$ & 0.346 \\
\hline CSM-3 & $2.910^{-6}$ & - & - & - \\
\hline ZW-01 & $1.610^{-7}$ & $-0.136 \pm 0.044$ & $1.36 \pm 0.05$ & 0.354 \\
\hline ZW-02 & $1.610^{-7}$ & $-0.153 \pm 0.044$ & $1.31 \pm 0.03$ & 0.350 \\
\hline ZW-07 & - & $-0.119 \pm 0.025$ & $1.36 \pm 0.05$ & 0.280 \\
\hline ZW-08 & - & $-0.084 \pm 0.018$ & $1.66 \pm 0.13$ & 0.375 \\
\hline ZW-09 & - & $-0.376 \pm 0.147$ & $1.45 \pm 0.11$ & 0.280 \\
\hline ZW-10 & - & $-0.272 \pm 0.086$ & $1.86 \pm 0.30$ & 0.320 \\
\hline
\end{tabular}

only the MO measurement. As a consequence, using only MO or only MT measurements allows for matching as good as possible with the van Genuchten model or the MO or MT measurement curve, but deviates too much from the MT or MO measurement. Hence, considering both measurements is advisable.

The hydraulic conductivity (Fig. $\mathbf{7 ( c )}$ ) and the water retention curve $(\mathbf{F i g .} \mathbf{7 ( d )})$ are presented for the MO and MT based parameters, together with the start-stop result from Kišon et al. (2013), and the average of CSM-1 and CSM-2 of Table 1. The higher $\gamma$ value for the centrifuge experiment relates in an upward shifted water retention curve. The gray area in these last two plots indicates the sampled saturations with the centrifuge. In other words, results outside the gray region are extrapolated values. In Fig. $\mathbf{7}(c)$, the curves for the start-stop result appear bad due to the saturated conductivity, which was determined to be much lower. In case $K_{s}=2.4 \times 10^{-6} \mathrm{~m} / \mathrm{s}$ would have been used in this figure, the result would have been comparable to the other presented curves. Furthermore, the hydraulic conductivity in Fig. $\mathbf{7 ( c )}$ for the ASTM data follows from the
SWCC by the Mualem theory, and was hence not directly tested, while for the centrifuge experiment, the conductivity has an important influence on the measurements.

We conclude that the relative unsaturated permeability can be recovered excellently, while the water retention curve has a good qualitative agreement (comparable $n$ parameter) with the previous CSM samples. We attribute this to sample preparation repeatability issues. The obtained relative permeability closely matches the values of Kišon et al. (2013), provided the same saturated conductivity is used.

\section{ZWIJNAARDE SAMPLES}

To be able to use higher rotational speeds, sensors which can withstand the higher weights are needed. The first tests use an outflow sensor with maximum $5 \mathrm{~kg}$-force (kgf) and hanging weight sensor with maximum $10 \mathrm{kgf}$. Accuracy is around $3 \mathrm{~g}$ for the first and $9 \mathrm{~g}$ for the second. This allows rotational speeds up to $1200 \mathrm{rpm}$. In later tests, we used a sensor of maximum $50 \mathrm{kgf}$ for the outflow and maximum $100 \mathrm{kgf}$ for the sample; accuracy is then around $22 \mathrm{~g}$ for the first and $30 \mathrm{~g}$ for the second. With

FIG. 6 Water retention curve in terms of (a) water content and in terms of (b) effective saturation for samples from the Zwijnaarde site. There are two samples per borehole.

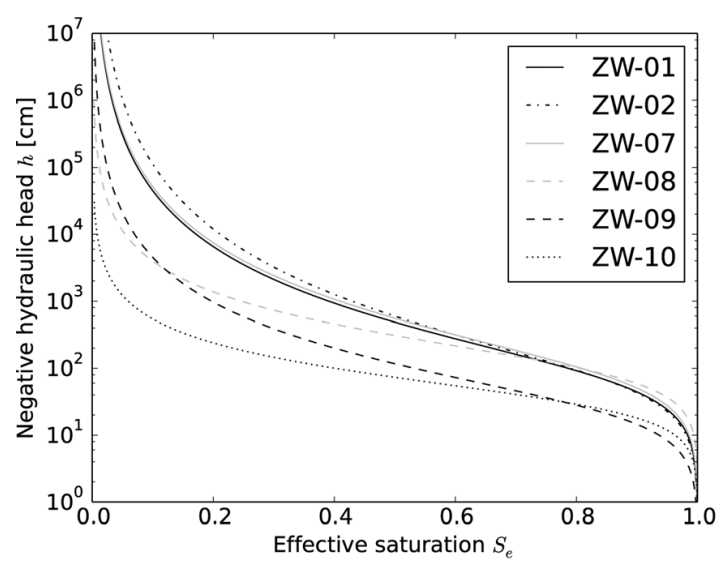

(a)

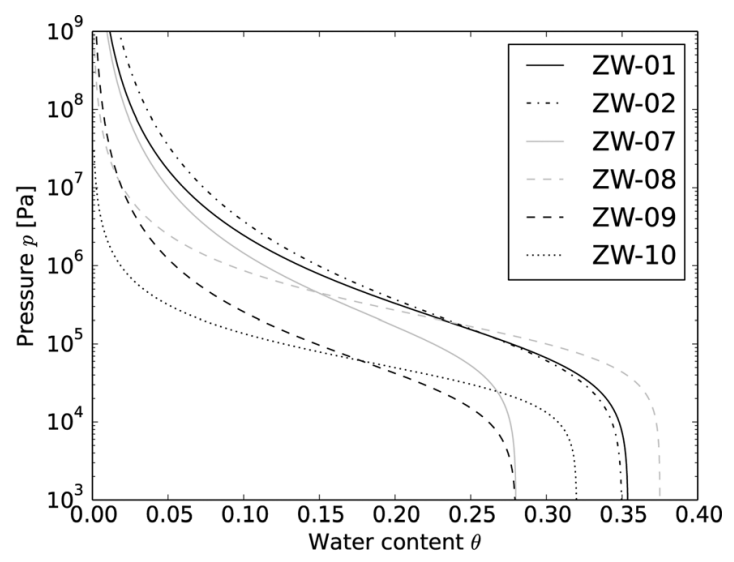

(b) 
TABLE 2 Results for CSM-3 with the centrifuge test.

\begin{tabular}{lccr}
\hline Sample & Method & $K_{s}\left(10^{-6} \mathrm{~m} / \mathrm{s}\right)$ & $\gamma(\mathrm{kPa})$ \\
\hline CSM-3 & Saturated & $2.4 \pm 0.1$ & - \\
CSM-3 & MO \& MT & - & - \\
CSM-3 & MO & - & $-0.052 \pm 0.006$ \\
CSM-3 & MT & - & $-0.040 \pm 0.001$ \\
\hline
\end{tabular}

these sensors, rotational speeds up to $4000 \mathrm{rpm}$ can be used. The weight of the outflow water is around $2 \mathrm{~g}$ in total (based on $60 \%$ drainage of a typical sample of $2.5 \mathrm{~cm}$ height). At $4000 \mathrm{rpm}$, this translates in an extra $6.2 \mathrm{kgf}$ on the sensor, while the tare of outflow holder will be $43.5 \mathrm{kgf}$ at that speed.

\section{Saturated Conductivity With the Centrifuge}

First, the saturated conductivity of the samples was determined in the centrifuge. The same sample, ZW-03, was tested twice. Due to the centrifugal force, some consolidation occurs, influencing the saturated conductivity. This can be readily investigated. During the first run of $20 \mathrm{~min}$, the sample contracted $3.8 \%$, from 2.11 to $2.03 \mathrm{~cm}$. During the second run of 5 min, another $1.7 \%$ decrease occurred.

As a consequence of this consolidation, the saturated conductivity slightly changes over the centrifuge run. This is visible in Fig. 8, which depicts the measurements during the first run of $20 \mathrm{~min}$. The measurement curve does not follow the optimal computed curve, which corresponds to a fixed $K_{s}$, as $K_{s}$ is here variable in reality.

FIG. 7 Clay Sand Mixture. Top: (a) Force applied by the expelled water, (b) force applied by the water in the sample tube. Crosses are the measured values, while the lines are the result of the parameters of Table $\mathbf{2}$ used in the model (so model predicted outflow). Bottom: (c) Resulting hydraulic conductivity and (d) water retention curve for the same parameter values. ASTM 1-5 are results with the parameters obtained by the pressure plate experiment, ASTM D2325 and $K_{s}=2.9 \times 10^{-6} \mathrm{~m} / \mathrm{s}$ start-stop is the result from Kišon et al. (2013).

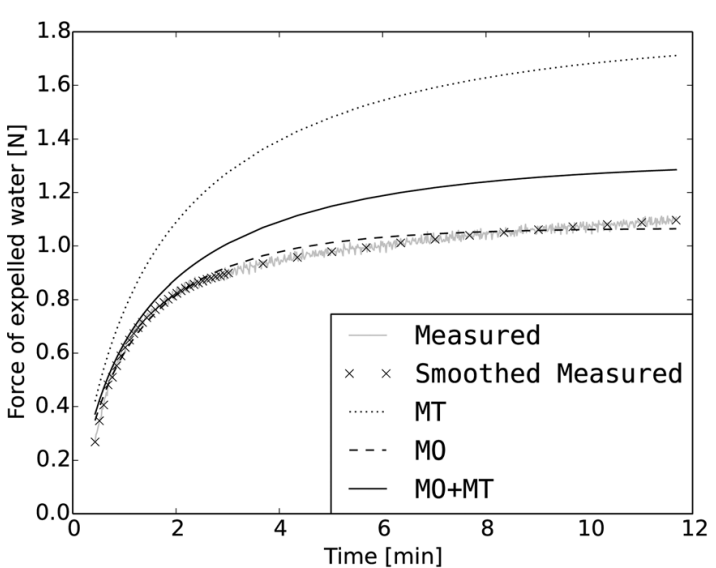

(a)

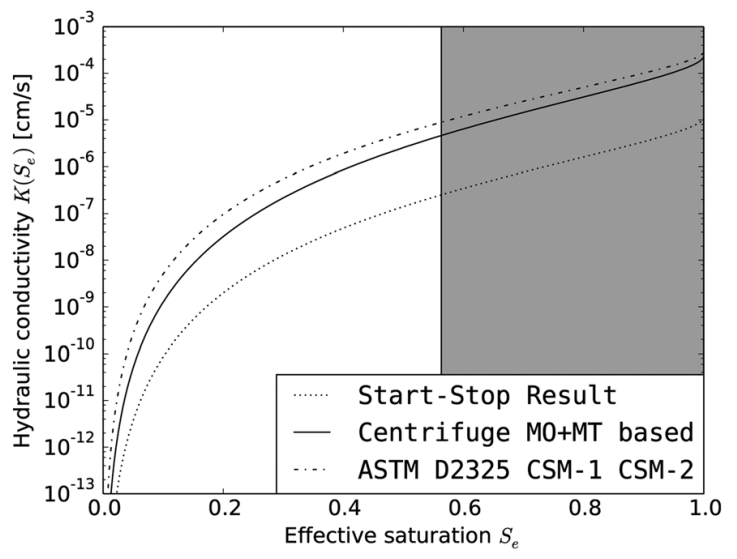

(c)

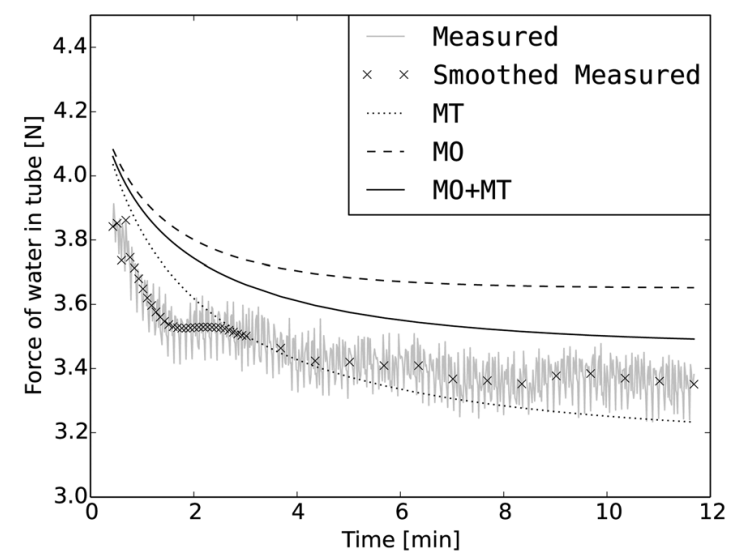

(b)

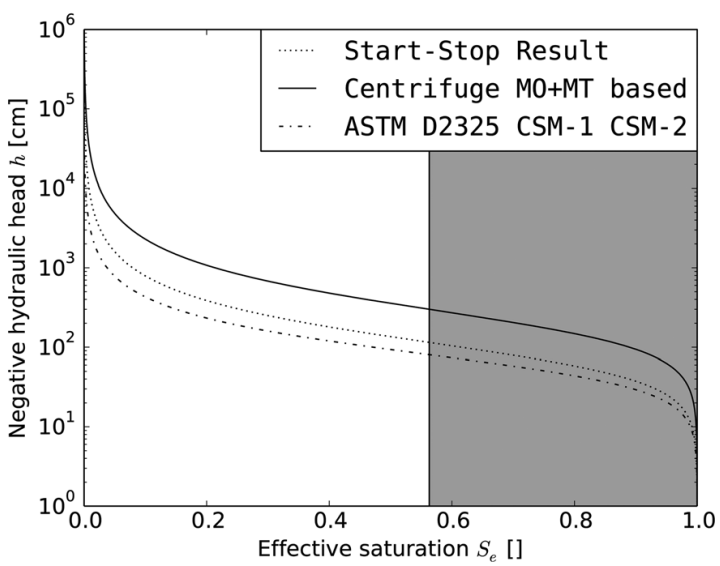

(d) 
FIG. 8 (a) Measurement weight on outflow sensor (MO), data points used after smoothing, and computed result. (b) Same for the sample tube weight (MT).

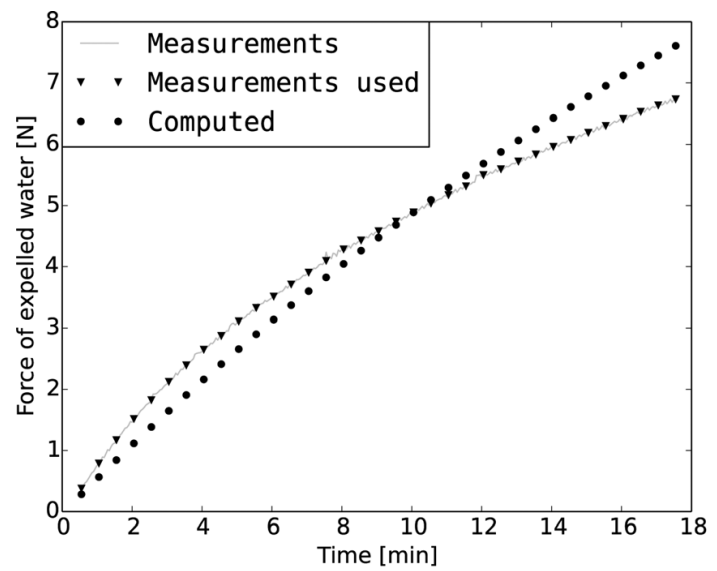

(a)

Using the centrifuge, $K_{s}$ can be determined from the sample tube weight (MT), from the outflow holder weight (MO), or from both combined (MO $+\mathrm{MT})$. It is also possible to only use measurements over a specific time interval. The results are depicted in Table 3. The results as obtained on some other samples have been added to the same tables. We can see that there is a variation in the order of one magnitude between the samples, with ZW-03 least conductive, and ZW-05 and ZW-06 more conductive. For sample ZW-03, some time was needed at $600 \mathrm{rpm}$ before the conductivity reached an equilibrium value around $1.6 \times 10^{-7} \mathrm{~m} / \mathrm{s}$.

\section{5 and 10 kgf Sensors}

Rotation started at $600 \mathrm{rpm}$ on sample ZW-03. After it was observed, only a little water weight was registered by the outflow sensor, and as there was no longer an increase in weight after $90 \mathrm{~min}$, the rotational speed was increased to $1000 \mathrm{rpm}$. During this, the tube weight sensor blocked as a vibration caused the weight to exceed the maximum of $10 \mathrm{kgf}$. As a consequence, the recovery of the water retention curve was based on the outflow weight and a part of the sample weight. The resulting soil parameters are given in Table 4. To have a good understanding of the results, a comparison of the water retention curve and the resulting conductivity is given in Fig. 9.

Note that it is required to include the measurements at $1000 \mathrm{rpm}$ to obtain this result. Using only the measurements (MO and MT) of the $600 \mathrm{rpm}$ phase, we are not able to determine the water retention curve. Too little water drained from the sample. Using only the MO data already gives good results in this case, which indicates that using the MT data of the $600 \mathrm{rpm}$ part only does not change the MO obtained result much. This experiment showed that it is important to have sensors adapted to the expected weights.

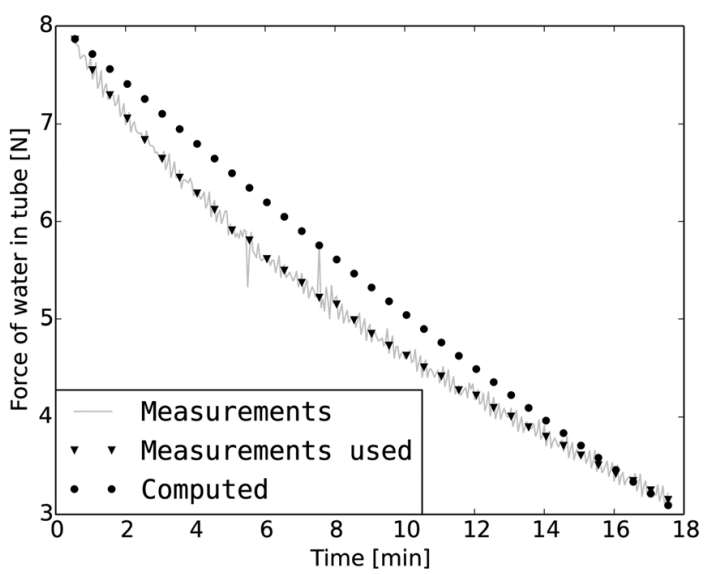

(b)

\section{0-100 kgf Sensors}

As for the Zwijnaarde sample, speeds of $1000 \mathrm{rpm}$ are required, but as the used low capacity sensors cannot handle this, higher capacity sensors were installed. Tests were done on sample $\mathrm{ZW}-04$. The sensors have $50 \mathrm{kgf}$ maximum capacity for outflow weight (MO) and $100 \mathrm{kgf}$ maximum capacity for the sample tube weight (MT). As indicated, the accuracy of these sensors is lower. Increasing the weight of the sample and outflow holder was required to reach the minimum value of the sensors (10\% of the maximum weight). Runs at $600 \mathrm{rpm}$ would not lead to usable data; as such, the sample was immediately rotated at $1000 \mathrm{rpm}$ and the first $20 \mathrm{~min}$ used. To even out the scatter due to the reduced accuracy, more aggressive smoothing was

TABLE 3 Determined saturated conductivity for ZW samples based on different analysis of the saturated centrifuge runs. Result and deviation are determined from a non-linear least-squares fit of the centrifuge model to the measurement. Based on the sample height decrease, average porosity $\theta_{s}$ is estimated. All tests are at $600 \mathrm{rpm}$ except if indicated otherwise.

\begin{tabular}{|c|c|c|c|c|c|}
\hline Description & Exp & $\begin{array}{l}\text { Time Used } \\
\quad(\min )\end{array}$ & $\begin{array}{c}K_{s} \\
\left(10^{-7} \mathrm{~m} / \mathrm{s}\right)\end{array}$ & $\begin{array}{c}\text { Deviation } \\
\left(10^{-7} \mathrm{~m} / \mathrm{s}\right)\end{array}$ & Est. $\theta_{s}$ \\
\hline $\mathrm{MO}+\mathrm{MT} \mathrm{ZW}-03$ & 1 & $0-3$ & 3.87 & 0.08 & 0.37 \\
\hline $\mathrm{MO}+\mathrm{MT} \mathrm{ZW}-03$ & 1 & $0-18$ & 2.77 & 0.04 & 0.355 \\
\hline $\mathrm{MO}+\mathrm{MT} \mathrm{ZW}-03$ & 1 & $12-18$ & 1.57 & 0.07 & 0.345 \\
\hline $\mathrm{MO}+\mathrm{MT} \mathrm{ZW}-03$ & 2 & $0-5$ & 1.57 & 0.06 & 0.34 \\
\hline MO ZW-03 & 2 & $0-5$ & 1.45 & 0.02 & 0.34 \\
\hline MT ZW-03 & 2 & $0-5$ & 1.95 & 0.06 & 0.34 \\
\hline MO ZW-04 & 4 & $4-7$ & 2.5 & 0.5 & - \\
\hline MO ZW-04 1000 rpm & 6 & $0-3.5$ & 3.1 & 0.1 & - \\
\hline MO ZW-05 & 1 & $0-1.5$ & 31.3 & 1.9 & - \\
\hline MO ZW-05 & 2 & $0-2.5$ & 8.9 & 0.15 & - \\
\hline MO ZW-06 & 3 & $0-1$ & 42.0 & 11.7 & - \\
\hline
\end{tabular}


TABLE 4 Resulting parameters arising in the model. If deviation is given, the parameter was determined via nonlinear least-squares, if not given, it was known input.

\begin{tabular}{lccr}
\hline Description & $K_{s}\left(10^{-7} \mathrm{~m} / \mathrm{s}\right)$ & $\gamma\left(\mathrm{kPa}^{-1}\right)$ & $\theta_{s}$ \\
\hline ZW-01 ASTM D2325 & - & $-0.136 \pm 0.044$ & $1.36 \pm 0.05$ \\
ZW-02 ASTM D2325 & - & $-0.153 \pm 0.044$ & $1.31 \pm 0.03$ \\
ZW-03 sat MO + MT & $1.6 \pm 0.04$ & - & -354 \\
ZW-03 MO & 1.6 & $-0.185 \pm 0.004$ & 0.350 \\
ZW-03 MO, part MT & 1.6 & $-0.167 \pm 0.004$ & 0.34 \\
\hline
\end{tabular}

FIG. 9 Experiment on ZW-03. (a) Water retention curves obtained. (b) Resulting hydraulic conductivity taking $K_{s}$ into account. In both cases comparison is made with the pressure plate ASTM experiment on ZW-01 and ZW-02

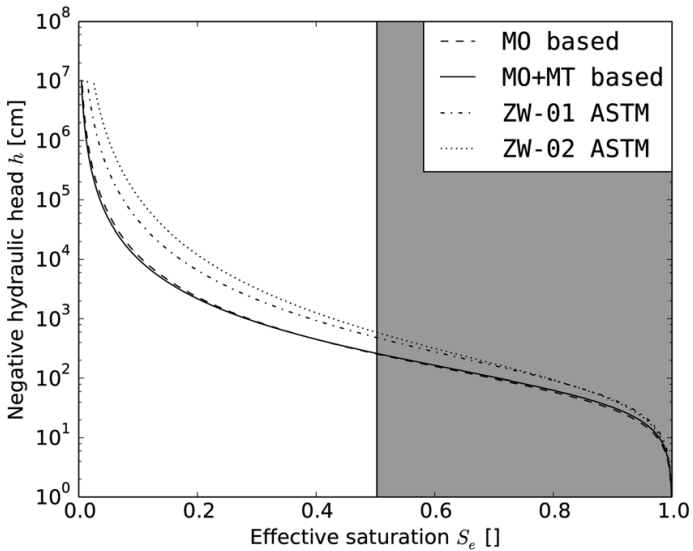

(a)

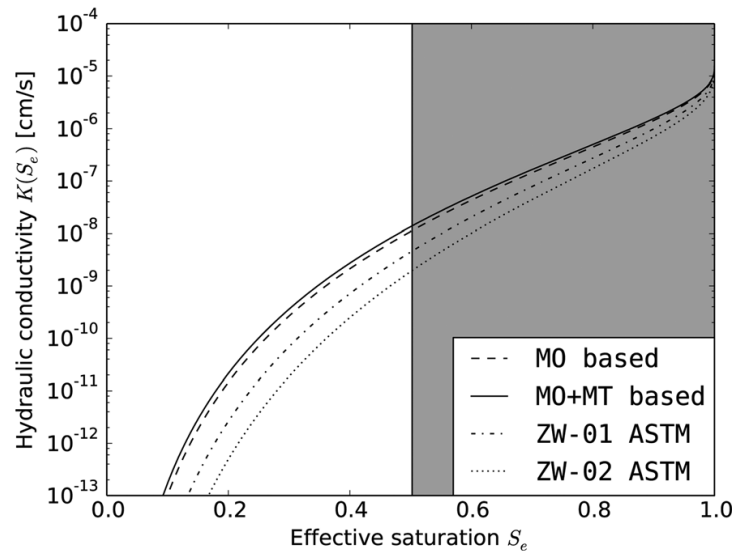

(b)

applied to the measurement (Gaussian smoothing with degree 25)

As at this rotational speed, we can expect a relatively large outflow during the ramp up of the centrifuge, but data acquired during this ramp up cannot be used in the model due to lack of a dedicated RPM sensor. We therefore expect the resulting SWCC and hydraulic conductivity to be less accurate at higher values of effective saturation. Indeed, using the previously determined $K_{s}$ of $3.1 \times 10^{-7} \mathrm{~m} / \mathrm{s}$, the remaining soil parameters were recovered with the results given in Table 5 . The corresponding water retention curve is given in Fig. 10. The recovered water

retention curve parameters are not as good as with $\mathrm{ZW}-03$, with especially $\gamma$ shifted. However, in the sampled saturation range (gray area in the figure), this does lead to a water retention curve that corresponds acceptably to the earlier experiments, especially in the range $S_{e} \in(0.6,0.8)$. We can conclude that the higher capacity sensors allow for an increase in the rotational speed, leading to comparable results as the low capacity sensors. Increasing the accuracy of the high capacity sensors should lead to comparable results as with $\mathrm{ZW}-03$ as the more aggressive smoothing would not be needed and the ramp-up data would be usable for the parameter identification.

TABLE 5 Resulting parameters arising in the model. If deviation is given, the parameter was determined via nonlinear least-squares, if not given, it was known input.

\begin{tabular}{lcrr}
\hline Description & $K_{s}\left(10^{-7} \mathrm{~m} / \mathrm{s}\right)$ & $\gamma\left(\mathrm{kPa}^{-1}\right)$ & $n$ \\
\hline ZW-01 ASTM D2325 & - & $-0.136 \pm 0.044$ & $\theta_{s}$ \\
ZW-02 ASTM D2325 & - & $-0.0153 \pm 0.044$ & 0.354 \\
ZW-03 & $1.48 \pm 0.06$ & - & 0.350 \\
ZW-04 & $3.1 \pm 0.1$ & - & -0.05 \\
ZW-03 MO, 20 min MT & 1.6 & $-0.167 \pm 0.004$ & - \\
ZW-04 MO + MT & 3.1 & $-0.068 \pm 0.003$ & 0.03 \\
\hline
\end{tabular}


FIG. 10 Water retention curves obtained for ZW-04 compared to the earlier results.

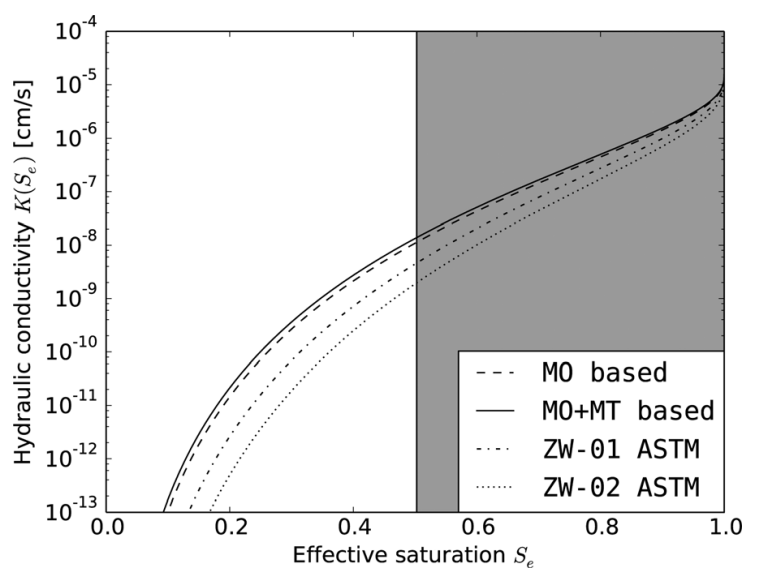

\section{Conclusion}

We presented an experimental method with the potential of determining the water retention curve and relative permeability in a matter of hours in the wet to moderately dry region for common soils. This is achieved by in flight transient measurements of two weights: Sample weight and outflow weight. The method does not disturb the sample and can be applied to all samples that undergo no deformation in a centrifuge. The model can be readily extended to also perform a freeform optimization of the water retention curve or apply another model than the van Genuchten-Mualem approach used here.

Further research is needed to determine how to handle samples that undergo contraction in the centrifuge during unsaturated flow. In addition, optimal techniques are needed to select rotational speeds (and change corresponding weight sensors) to allow the determination of the SWCC in the entire range of $S_{e} \in(0.4,1)$ where centrifuge fluid flow corresponds with normal fluid flow.

\section{ACKNOWLEDGMENTS}

This research project is funded by IWT (Agency for Innovation by Science and Technology) and Geosound.be, within the project CENPERON (Centrifuge for the determination of the permeability of unsaturated soils), IWT project 110317.

\section{References}

ASTM D6836-00, Standard Test Methods for Determination of the Soil-Water Characteristic Curve for Desorption Using a Hanging Column, Pressure Extractor, Chilled Mirror Hygrometer, and/or Centrifuge, ASTM International, West Conshohocken, PA, 2008, www.astm.org.

Caputo, M. C. and Nimmo, J. R., 2005, "Quasi-Steady Centrifuge Method for Unsaturated Hydraulic Properties," Water Resour. Res., Vol. 41, pp. 1-5.
Chiu, T.-F. and Shackelford, C. D., 1998, "Unsaturated Hydraulic Conductivity of Compacted Sand-Kaolin Mixtures," J. Geotech. Geoenviron. Eng., Vol. 124, No. 2, pp. 160-170.

Conca, J. L. and Wright, J. V., 1998, "The UFA Method for Rapid, Direct Measurements of Unsaturated Transport Properties in Soil, Sediment and Rock," Aust. J. Soil Res., Vol. 36, No. 2, pp. 291-316.

Firoozabadi, A. and Aziz, K., 1991, "Relative Permeabilities From Centrifuge Data," J. Can. Petrol. Technol., Vol. 30, No. 5, pp. 33-42.

Hassler, G. L. and Brunner, E., 1945, "Measurements of Capillary Pressure in Small Core Samples," Trans. AIME, Vol. 160, No. 1, pp. 114-123.

Kačur, J., Malengier, B., and Kišon, P., 2011, "Using Global Characteristics of a Centrifuge Outflow Experiment to Determine Unsaturated Soil Parameters," Math. Probl. Eng., Vol. 2011, p. 163020.

Khaleel, R., Relyea, J., and Conca, J., 1995, "Evaluation of van Genuchten-Mualem Relationships to Estimate Unsaturated Hydraulic Conductivity at Low Water Contents," Water Resour. Res., Vol. 31, No. 11, pp. 2659-2668.

Kišon, P., Malengier, B., Di Emidio, G., Peiffer, H., and Van Keer, R., 2013, "Inverse Determination of Saturated and Relative Permeability With a Bench-Scale Centrifuge," Inverse Problems in Science and Engineering, Vol. 23, No. 1, pp. 16-37.

Martins Reis, R., Nogueira Sterck, W., Bastos Ribeiro, A., Dell'Avanzi, E., Saboya, F., Tibana, S., Roberto Marciano, C., and Ramires Sobrinho, R., 2011, "Determination of the Soil-Water Retention Curve and the Hydraulic Conductivity Function Using a Small Centrifuge," Geotech. Test. J., Vol. 34, No. 5, pp. 457-466.

McCartney, J. S. and Zornberg, J. G., 2010, “Centrifuge Permeameter for Unsaturated Soils. II: Measurement of the Hydraulic Characteristics of an Unsaturated Clay," J. Geotech. Geoenivron. Eng., Vol. 136, No. 8, pp. 1064-1076.

Nakajima, H. and Stadler, A. T., 2006, "Centrifuge Modeling of One-Step Outflow Tests for Unsaturated Parameter Estimations," Hydrol. Earth Syst. Sci. Discuss., Vol. 3, pp. 731-768.

Nimmo, J. R., 1990, "Experimental Testing of Transient Unsaturated Flow Theory at Low Water Content in a Centrifugal Field," Water Resour. Res., Vol. 26, No. 9, pp. 1951-1960.

Nimmo, J. R., Rubin, J., and Hammermeister, D. P., 1991, "Unsaturated Flow in a Centrifugal Field: Measurement of Hydraulic Conductivity and Testing of Darcy's Law," Water Resour. Res., Vol. 27, No. 6, pp. 1263-1269.

Nimmo, J. R., Perkins, K. S., and Lewis, A. M., 2002, "SteadyState Centrifuge," Methods of Soil Analysis, Part 4, Soil Science Society of America, Inc, Madison, WI, pp. 903-916.

Parks, J. M., Stewart, M. A., and McCartney, J. S., 2012, "Validation of a Centrifuge Permeameter for Investigation of Transient Infiltration and Drainage Flow Processes in Unsaturated Soils," Geotech. Test. J., Vol. 35, No. 1, GTJ103625, pp. 182-192.

Peters, A. and Durner, W., 2008, "Simplified Evaporation Method for Determining Soil Hydraulic Properties," J. Hydrol., Vol. 356, Nos. 1-2, pp. 147-162.

Schelle, H., Iden, S. C., and Durner, W., 2011, "Combined Transient Method for Determining Soil Hydraulic Properties in a Wide Pressure Head Range," Soil. Sci. Soc. Am. J., Vol. 75, No. 5, pp. 1681-1693. 
Van den Berg, E. H., Perfect, E., Tu, C., Knappett, P. S. K., Leao, T. P., and Donat, R., 2009, "Unsaturated Hydraulic Conductivity Measurements With Centrifuges: A Review," Vadose Zone J., Vol. 8, No. 3, pp. 531-547.

van Genuchten, M. T., 1980, "A Closed-Form Equation for Predicting the Hydraulic Conductivity of Unsaturated Soils," Soil Sci. Soc. Am. J., Vol. 44, No. 5, pp. 892-898.
Šimůnek, J. and Nimmo, J. R., 2005, "Estimating Soil Hydraulic Parameters From Transient Flow Experiments in a Centrifuge Using Parameter Optimization Technique," Water Resour. Res., Vol. 41, WR003379.

Zornberg, J. G. and McCartney, J. S., 2010, "Centrifuge Permeameter for Unsaturated Soils. I: Theoretical Basis and Experimental Developments," J. Geotech. Geoenviron. Eng., Vol. 136, No. 8, pp. 1051-1063. 